\title{
BMJ Open Government management capacities and the containment of COVID-19: a repeated cross-sectional study across Chinese cities
}

\author{
Wenchao Li, ${ }^{1}$ Jing Li (D) , ${ }^{2}$ Junjian $\mathrm{Yi}^{3,4}$
}

To cite: Li W, Li J, Yi J. Government management capacities and the containment of COVID-19: a repeated cross-sectional study across Chinese cities. BMJ Open 2021;11:e041516. doi:10.1136/ bmjopen-2020-041516

- Prepublication history and additional supplemental materials for this paper are available online. To view these files, please visit the journal online (http://dx.doi.org/10. 1136/bmjopen-2020-041516)

Received 11 June 2020

Revised 13 November 2020 Accepted 10 February 2021

D) Check for updates

(c) Author(s) (or their employer(s)) 2021. Re-use permitted under CC BY-NC. No commercial re-use. See rights and permissions. Published by BMJ.

${ }^{1}$ School of Economics and Management, Tongji University, Shanghai, China

${ }^{2}$ School of Economics,

Singapore Management University, Singapore

${ }^{3}$ Department of Economics, The Chinese University of Hong Kong, Hong Kong, China ${ }^{4}$ Department of Economics, National University of Singapore, Singapore

Correspondence to Dr Jing Li; lijing@smu.edu.sg

\section{ABSTRACT}

Objectives Better understanding of the dynamics of the COVID-19 (2019 novel coronavirus disease) pandemic to curb its spread is now a global imperative. While travel restrictions and control measures have been shown to limit the spread of the disease, the effectiveness of the enforcement of those measures should depend on the strength of the government. Whether, and how, the government plays a role in fighting the disease, however, has not been investigated. Here, we show that government management capacities are critical to the containment of the disease.

Setting We conducted a statistical analysis based on cross-city comparisons within China. China has undergone almost the entire cycle of the anticoronavirus campaign, which allows us to trace the full dynamics of the outbreak, with homogeneity in standards for statistics recording. Primary and secondary outcome measures Outcome measures include city-specific COVID-19 case incidence and recoveries in China.

Results The containment of COVID-19 depends on the effectiveness of the enforcement of control measures, which in turn depends on the local government's management capacities. Specifically, government efficiency, capacity for law enforcement, and the transparency of laws and policies significantly reduce COVID-19 prevalence and increase the likelihood of recoveries. The organisation size of the government, which is not closely related to its capacity for management, has a limited role.

\section{INTRODUCTION}

COVID-19 outbreaks have raced around the world and have exploded into a pandemic. About 47.3 million infections have been confirmed in more than 200 countries and territories. It has become a global imperative to better understand the dynamics of this pandemic in order to limit its ongoing spread.

China, which was the first country exposed to the coronavirus, has almost completed the full cycle of the anticoronavirus campaign. Since mid-March 2020, daily new cases in China have been reduced to near-zero
Strengths and limitations of this study

- We are among the first to examine the role of government management capacities in the containment of COVID-19.

- We conducted a repeated cross-sectional study in China which ensures consistency in standards for statistics recording and homogeneity in institutional features.

- We were able to trace the full dynamics of the outbreak in the setting of Chinese cities.

- The specific measures of government management capacities may not be readily comparable with cities in other countries.

- The restrictive government disease control practices may also not be readily applicable to other countries.

levels (figure 1). This result is substantially attributed to the strict travel restrictions and containment measures, such as suspending public transport, closing entertainment venues and banning public gatherings, implemented by Chinese authorities. ${ }^{1-5}$ The WHO has repeatedly praised China for its effective response to the COVID-19 outbreak.

Yet China's response is not free of controversy, in particular whether the government and the measures it has taken have succeeded in fighting the disease. For instance, sceptics point out that other places, such as Singapore, imposed similar containment measures but still experienced an enormous outbreak. ${ }^{67}$

The effectiveness of the enforcement of control measures should depend on the strength of the government, as indicated by the notion of state capacity. State capacity is shown to be crucial to economic development and technological change. ${ }^{89}$ The rapid economic growth in East Asian economies, in particular, can largely be accounted for by states with a great deal of capacity. ${ }^{8}$ During this pandemic, heated discussions have centred on responses by different countries, 
A Daily new cases

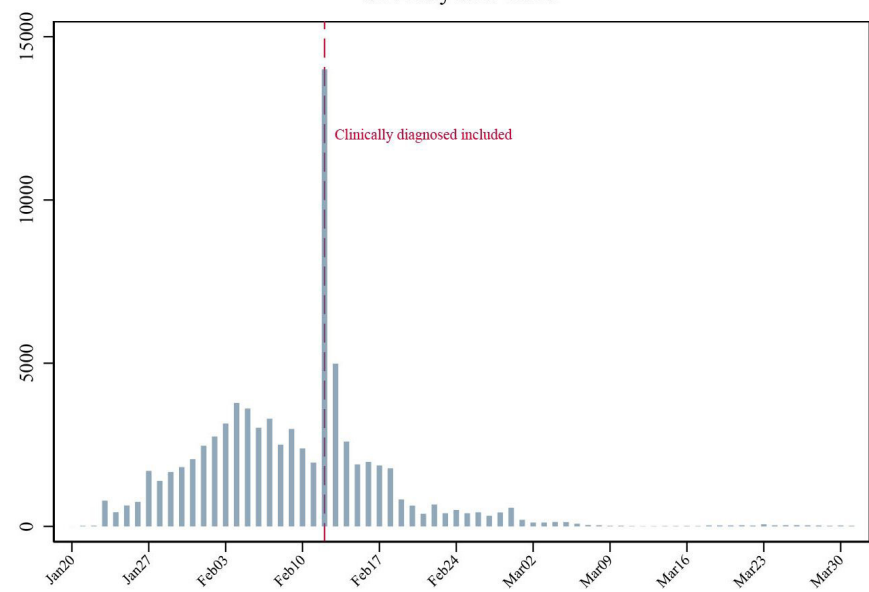

B Daily new recoveries

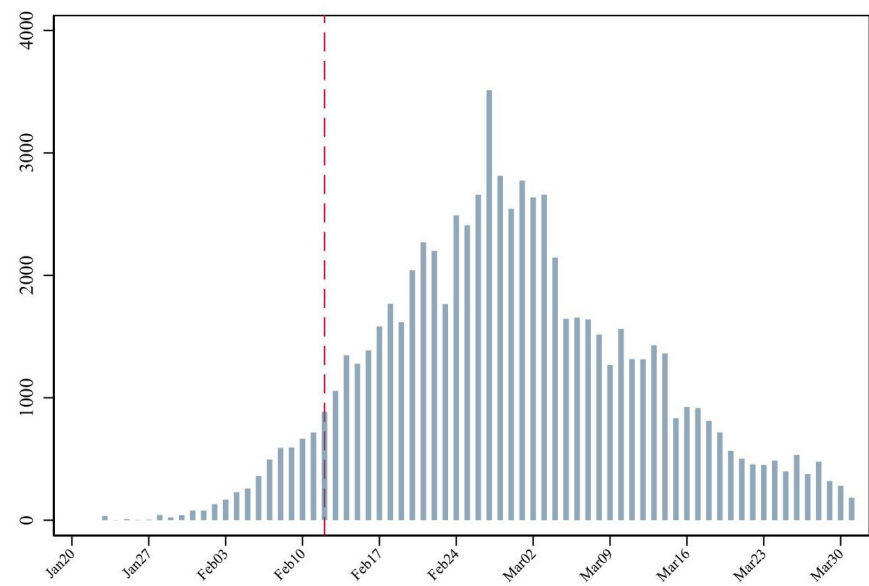

Figure 1 Daily (A) new cases and (B) recoveries in China, January-March. From 12 February on, new cases include clinically diagnosed cases, in addition to those confirmed by nucleic acid tests, for cities in Hubei Province. This results in a sharp increase in the number of daily new cases, as indicated by the vertical line in the first plot.

which are said to 'reveal the need for a strong state'. ${ }^{10}$ The discussions echo the notion of state capacity or government management capacity. Anecdotal evidence indicates that within China there are fewer COVID-19 cases in cities that implemented control measures more pre-emptively, ${ }^{1}$ which highlights the importance of the management capacity of local governments for containing the disease.

Whether, and how, the government plays a role in fighting the disease, however, has not been formally investigated. In this paper, we show that government management capacities are critical to the containment of the disease by conducting a statistical analysis based on cross-city comparisons within China. Because China has undergone almost the entire cycle of the anticoronavirus campaign, we can trace the full dynamics of the outbreak while being consistent in standards for statistics recording. We find that better government management-as measured by government efficiency, capacity for law enforcement, transparency of laws and policies, and an aggregate management index-is significantly associated with both reductions in case incidence and increases in recoveries. Government organisation size, in comparison, has an insignificant effect.

These findings demonstrate the important role of the government in controlling COVID-19 and thereby help political leaders and health authorities around the world better understand the dynamics of the pandemic. They also contribute to discussions of the need for strong states as revealed by the pandemic. ${ }^{10}$

\section{Government management capacity}

In epidemiology, compartmental models suggest that the implementation of effective public health measures lowers the infection rate and reduces the case incidence. ${ }^{11} 12$ The implementation of public health measures, in turn, is related to the notion of state capacity-or, more specifically, government management capacity-in economics. ${ }^{89}$ To examine the role of state capacity in the containment of COVID-19 in China, we draw a spectrum of measures from the 2019 Global Urban Competitiveness Yearbook:

- Government efficiency measures administrative procedures and time lags in the local government's functions.

- Capacity for law enforcement measures the local government's ability to enforce the rule of law.

- Transparency of laws and policies measures how well laws and policies stipulated by the local government are known to citizens.

- Government organisation size refers to the number of employees in government agencies and organisations as a percentage of total population.

- An aggregate government management index measures the overall management level and public policy environment of a city.

The four subindicators and the aggregate index are closely related to the management capacity of local governments. All of them are on a 10-100 scale. A large value indicates better management of the local government (see online supplemental file 1 for details about the construction of these measures).

\section{Study design}

Our study design is based on three unique contextual features. First, as noted earlier, China is in the final stage of the COVID-19 outbreak, which allows us to trace the full dynamics of the outbreak. Second, a within-country analysis ensures homogeneity in the national response, institutional background, and more importantly standards for COVID-19 statistics recording. Third, China banned travel to and from Wuhan City-the epicentre of the outbreak-on 23 January 2020. The ban impeded the growth and limited the size of the epidemic elsewhere in the country, and as a result allowed local governments to undertake effective control measures. ${ }^{5}$

Specifically, we conducted a statistical analysis in which we exploit variations in a spectrum of city-specific government management capability measures and examine how those variations are linked to variations in the effectiveness 
of COVID-19 control. According to the Ministry of Civil Affairs, there are 333 prefecture-level cities in China, which include prefectures, municipalities, provincial county-level cities and subprovincial cities (special administrative regions, Hong Kong and Macau, and Taiwan are excluded). We included 332 in our sample and excluded the epicentre, Wuhan City. The sample spans a period of 3 months, from January to late March. This period immediately followed the Wuhan lockdown, when local governments began to implement various measures to curb the further spread of infections. ${ }^{1}$ We use ordinary least squares regressions to examine the effects of government management in different phases of the outbreak, on a weekly basis. We carried out the statistical analysis using Stata V.16.

In the regression models, outcome variables are the number of new cases and the number of new recoveries in a city. Those numbers have been recorded daily by the National Health Commission of China since January 2020, which we aggregate into weekly data. Recovery rate, defined as the cumulative total recoveries over the cumulative number of closed cases (recoveries plus deaths), has been more than $95 \%$.

Explanatory variables include a set of city-specific, time-invariant determinants of the spread and control of COVID-19. We are particularly interested in the above-mentioned government management capability measures. We also examine other important determinants: population age structure, connection with Wuhan and the local health system's capacity. Data on population age structure (elderly, children and working-age population as a percentage of total population) are from the 2015 China population mini-census. Based on an index of the size of daily population flow that proxies for the total intensity of migration out of Wuhan to other cities (provided by Baidu Migration), we constructed a variable by calculating the average of the migration index over 14 days before the lockdown of Wuhan. We also considered the share of Wuhan-origin residents in the city, using data from the census. Health system capacity is proxied by the total number of hospital beds in the city and the total number of hospital employees, based on data from the 2019 China City Statistical Yearbook (see Extended data table 1 in online supplemental file 1 for definitions and summary statistics of these variables).

\section{Patient and public involvement}

No patients were involved in this research.

\section{RESULTS}

Figure 2 displays the estimated coefficients on the key determinants from regressions of the number of new cases (in panel A) and new recoveries (in panel B) in a week at the city level. The orange vertical bars are the $90 \%$ CIs. For example, the first bar in each plot of panel

A Weekly new cases
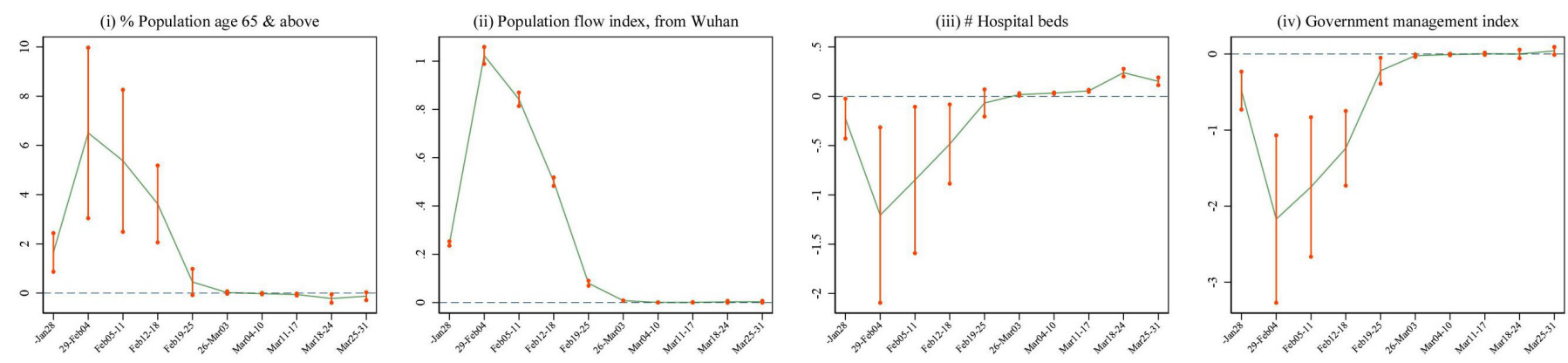

B Weekly new recoveries
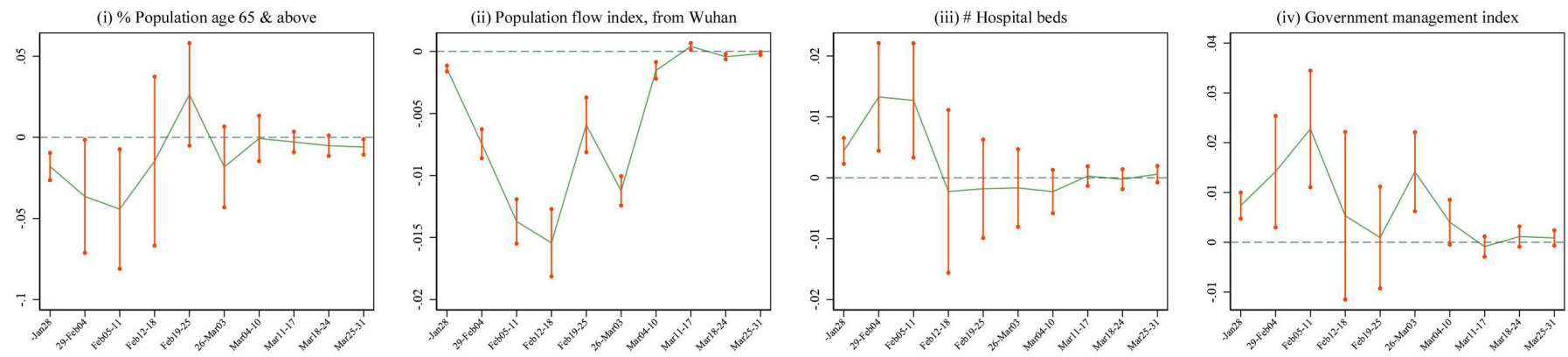

Figure 2 Coronavirus cases and recoveries: key determinants. Each panel displays the estimated coefficients from the regressions of weekly number of $(A)$ new cases and $(B)$ recoveries within a city on four key determinants; $90 \%$ Cls are shown as orange vertical bars. All regressions control for percentage of population aged 65 and above, share of Wuhan-origin residents (or an index of population flow from Wuhan), total population, employment rate, percentage of population with a college degree and an indicator variable for municipality. In panel B, regressions additionally control for the number of closed cases. 
A regresses the number of cases for the week ending 28 January on city-level factors. The second bar regresses the number of cases for the week ending 4 February on the same city-level factors, and so on. Additional control variables include the share of Wuhan-origin residents, total population, employment rate, percentage of population with a college degree and an indicator variable for municipality. For recoveries, regressions also control for the number of closed cases in that week. Conditional on those variables, the estimated coefficients from the regressions reflect the effects of the determining factors of our interest during the coronavirus outbreak.

\section{Population age structure}

As the first column of figure 2 shows, the proportion of people aged 65 and above is closely related to the morbidity and mortality of COVID-19, especially in the initial phase of the outbreak. Specifically, a larger share of the elderly in the local population is associated with more confirmed cases and fewer recoveries. This is in line with our expectation that the elderly have higher COVID-19 infection and death rates, and more elderly predict a larger chance of infection among high-risk populations. In panel $\mathrm{A}$, the small negative effects at the tail of the curve are in line with the interpretation that the elderly realise that they are particularly vulnerable and therefore pay closer attention to protecting themselves from the virus (see tables S1-1 to S1-10 in online supplemental file 2 for regression results). We also verify that normalising the aggregate variables, such as the number of new cases, the number of new recoveries and the number of hospital beds, by city-level population size yields similar patterns of results, as shown in Extended data figure 1 in online supplemental file 1.

In comparison, the share of children (age 0-15) does not have a clear relationship with the spread of the virus; the share of the working-age population (age 16-55) is negatively associated with the number of new cases and positively associated with the number of recoveries, as expected (see Extended data figure 2 in online supplemental file 1 and tables S2-1 to S2-10 in online supplemental file 2).

\section{Connection with the epicentre}

The second column of figure 2 indicates that a connection with Wuhan is a crucial determining factor. A larger index of population flow from Wuhan to a destination city is associated with more infected cases and fewer recoveries. This finding corresponds with the interpretation that population flow out of the epicentre of the outbreak increases the likelihood that people who are infected will come into contact with people who are not. We observe that the effects on new cases vanish at the end of February, while the effects on recoveries appear strong across different stages of the outbreak and last to mid-March.

\section{Health system capacity}

The third column of figure 2 shows that during the early phases of the outbreak, health system capacity—as proxied by the total number of hospital beds-is negatively associated with the number of new cases and positively associated with the number of recoveries. A local health system's capacity to effectively admit those who are already infected is crucial to reducing transmission among residents, and the capacity to respond to the needs of the infected, who often require admission to an intensive care unit, is vital to increasing the chance of recovery.

During the later phases of the outbreak, however, the effects of health system capacity become insignificant. At the end of March, the effects on new cases turn positive, which is partly due to China's patient reallocation strategy: the central government transferred some patients from cities where local health systems were overwhelmed to nearby cities with greater availability of medical resources. ${ }^{13}$ However, this move may result in more coronavirus transmission in destination cities. (Using the number of hospital employees as a proxy for health system capacity yields similar conclusions; see Extended data figure 2 in online supplemental file 1 and tables S2-1 to S2-10 in online supplemental file 2.)

\section{Government management index}

The last column of figure 2 shows that the government management index is an important determining factor of the spread and control of COVID-19. Better government management is significantly associated with reductions in case incidence, with the largest effect observed from early through mid-February, when the outbreak was at its peak. This pattern is similar to the effects of local health system capacity shown in the third column of panel A. The small positive effect at the end of March can also be attributed to the patient reallocation strategy, whereby patients tend to be transferred to cities with better government management.

In addition, better government management is associated with increases in the weekly number of recoveries conditional on the number of closed cases, as panel B shows. The effects appear to be the largest in midFebruary and are stronger and longer lasting than the effects of health system capacity. We further divide the cities into subgroups based on four criteria and conduct a series of subgroup analyses. We discuss the results in online supplemental file 1 under the 'Subgroup analysis' section (see Extended data figures 3-6 in online supplemental file 1 for results).

While governments with better functions, such as higher government efficiency and transparency of laws, can implement better disease control measures and thereby reduce new cases and increase recovery cases, other pathways of the effects may exist. For instance, it is possible that governments with higher transparency are more accurate in releasing case numbers, which might lead to a larger number of new cases and a smaller number of recovery cases. This possibility, however, does not substantially affect the interpretation of our results. The other pathway of the effects implies that the values of the outcome variables contain non-random measurement 
A Weekly new cases
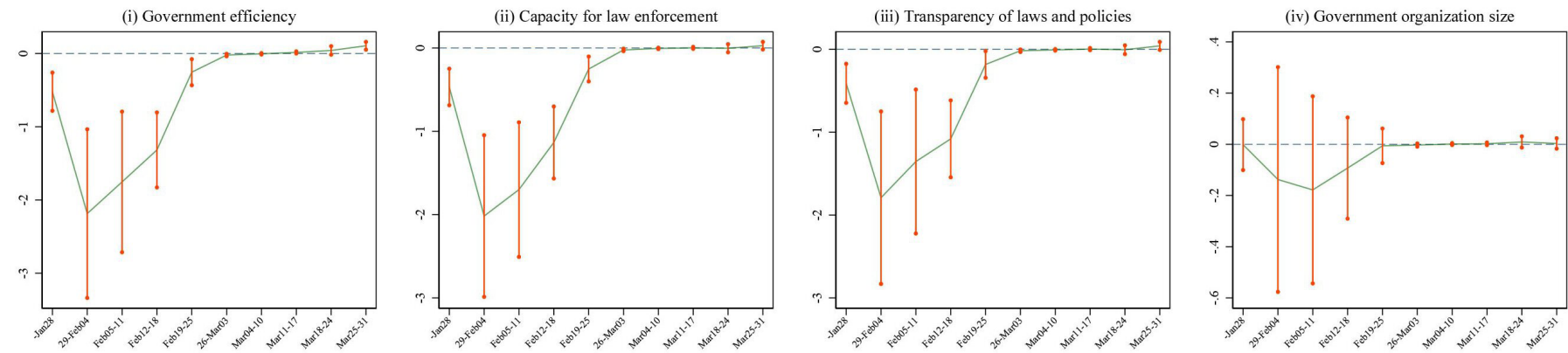

B Weekly new recoveries
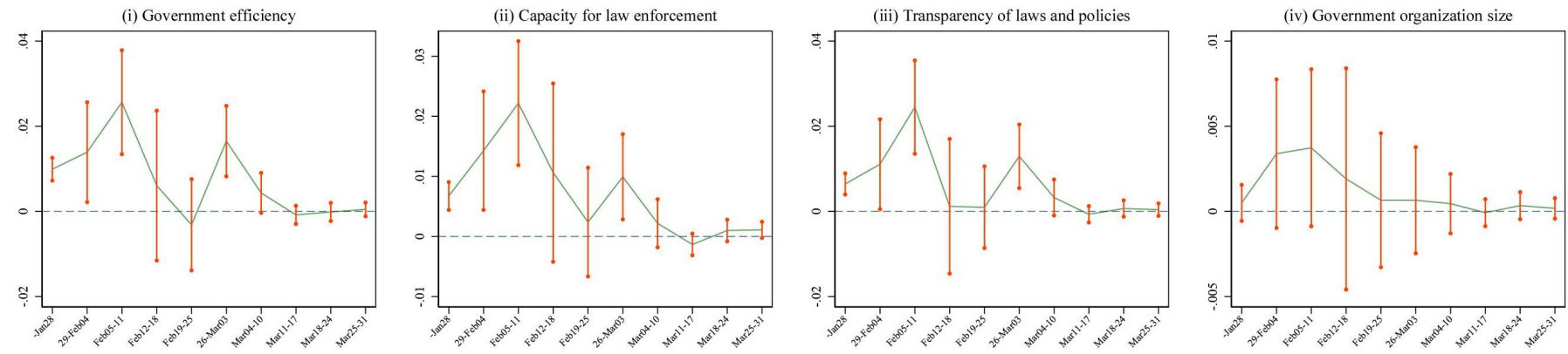

Figure 3 Coronavirus cases and recoveries: the role of government management. Each panel displays the estimated coefficients from the regressions of weekly number of $(A)$ new cases and $(B)$ recoveries within a city on four indicators of government management capabilities; $90 \%$ Cls are shown as orange vertical bars. All regressions control for percentage of population aged 65 and above, share of Wuhan-origin residents, total population, employment rate, percentage of population with a college degree and an indicator variable for municipality. In panel B, regressions additionally control for the number of closed cases.

errors, which would give rise to a downward bias in the estimations. Therefore, the effect we have identified in the statistical analysis at least represents a lower bound of the true effect of government management capacities on the disease control, which applies to both new and recovery cases.

To gain a more comprehensive understanding of the government's role in curbing the coronavirus outbreak, we further regress the number of new cases and recoveries on a weekly basis on the four subindicators of government management capabilities separately, plus a range of control variables.

Figure 3 displays the estimated coefficients on the subindicators and the CIs. We find that government efficiency, capacity for law enforcement, and transparency of laws and policies exhibit similar patterns of effects as the aggregate index; they are negatively related to the number of new cases and positively related to the number of new recoveries. The effects of government organisation size, in comparison, are insignificant in both new infections and new recoveries (see tables S3-1 to S3-10 in online supplemental file 2 for regression results).

For regressions of new cases, the R-squared ranges from 0.1 to 0.6 , showing a reasonably good goodness of fit. For regressions of new recoveries, the R-squared is high because we control for the number of closed cases (recoveries plus deaths) in the week. Especially towards the end of the period, there were fewer deaths and the number of recoveries was very close to the number of closed cases, which lead to high R-squared.

\section{DISCUSSION}

The patterns documented demonstrate that, in addition to demographic controls, the containment of COVID-19 critically depends on the effectiveness of the enforcement of control measures designed for this purpose, which in turn depends on the local government's management capacities. Specifically, government efficiency determines the local government's competence in implementing containment measures; capacity for law enforcement determines how well the government can strengthen and effectively enforce containment measures; and the transparency of laws and policies determines how interim measures are understood, supported and cooperated with by citizens. Therefore, they significantly reduce COVID-19 prevalence and increase the likelihood of recoveries. The organisation size of the government, which is not closely related to its capacity for management, has a limited role.

In addition, our results show that new recovery rates, which have a positive association with health system capabilities, also have a positive association with government management capacities. This is possibly because health system capabilities heavily depend on government 
functions, especiallyin China, where a large share of healthcare sectors are managed—and at least partly owned-by the government. Therefore, government management capacity would have an impact on the efficiency and effectiveness of healthcare and medical resource allocations, which in turn determine the treatment outcomes for infected patients. Indeed, we have seen from figure 2 that the graphical patterns of the effects of the proxy for health system capacity (the number of hospital beds) and the proxy for government management capability (the government management index) appear similar.

We now discuss the potential methodological limitations of our study. First, our study is based on observational data instead of experimental data. Unobservable heterogeneity across cities in, for example, hygiene and nutrition, is likely to be correlated with the capacity of the local government and at the same time has an impact on the containment of the disease. As a result, it is difficult to directly obtain rigorous causal inference from the regression analysis. Second, our findings are based on a repeated cross-sectional study across Chinese cities, which may lack external validity due to the potential differences across countries in terms of institutions and legal systems. Third, although we have provided some plausible explanations, we cannot identify the exact mechanisms through which government effectiveness plays a role in the containment of the disease-for instance, whether public health measures or restrictions of mobility are more critical. Last, while our study has shown that governments with higher management capacities could impose effective containment measures to reduce COVID-19 prevalence, it keeps silent on whether the measures are cost-effective. A further cost-benefit analysis might be needed to provide better policy suggestions.

\section{CONCLUSION}

We have discussed, and formally investigated, the role of government in the containment of COVID-19, based on cross-city comparisons within China. We show that government management capacities are vital to controlling the disease.

Our analysis neither speaks to the feasibility of specific containment measures-whether they can be replicated outside China-nor to the suitability-whether they are violations of human rights. ${ }^{14}$ With that caveat, our analysis shows that government management capacities are strongly associated with the containment of COVID-19. This study could help political leaders and health authorities around the world better understand the government's role in controlling the outbreak. In particular, governments that are slow and inefficient in response to the outbreak may contribute to its continuing spread worldwide. ${ }^{10}$ This could have important implications for future epidemics and public health emergencies.

Correction notice This article has been corrected since it was published. Affiliation for Junjian Yi has been corrected.
Contributors WL, JL and JY designed and performed the research, analysed the data and wrote the paper. All authors equally contributed to this research paper.

Funding The authors have not declared a specific grant for this research from any funding agency in the public, commercial or not-for-profit sectors.

Competing interests None declared.

Patient consent for publication Not required.

Provenance and peer review Not commissioned; externally peer reviewed.

Data availability statement Data are available upon reasonable request. All data relevant to the study are included in the article or uploaded as supplementary information. Extra data can be accessed via the Dryad Data Repository at http:// datadryad.org/ with doi:10.5061/dryad.q83bk3jh7.

Supplemental material This content has been supplied by the author(s). It has not been vetted by BMJ Publishing Group Limited (BMJ) and may not have been peer-reviewed. Any opinions or recommendations discussed are solely those of the author(s) and are not endorsed by BMJ. BMJ disclaims all liability and responsibility arising from any reliance placed on the content. Where the content includes any translated material, BMJ does not warrant the accuracy and reliability of the translations (including but not limited to local regulations, clinical guidelines, terminology, drug names and drug dosages), and is not responsible for any error and/or omissions arising from translation and adaptation or otherwise.

Open access This is an open access article distributed in accordance with the Creative Commons Attribution Non Commercial (CC BY-NC 4.0) license, which permits others to distribute, remix, adapt, build upon this work non-commercially, and license their derivative works on different terms, provided the original work is properly cited, appropriate credit is given, any changes made indicated, and the use is non-commercial. See: http://creativecommons.org/licenses/by-nc/4.0/.

ORCID iD

Jing Li http://orcid.org/0000-0001-5269-134X

\section{REFERENCES}

1 Tian H, Liu Y, Li Y, et al. An investigation of transmission control measures during the first 50 days of the COVID-19 epidemic in China. Science 2020;368:abb6105

2 Kraemer MUG, Yang C-H, Gutierrez B, et al. The effect of human mobility and control measures on the COVID-19 epidemic in China. Science 2020;368:abb4218

3 Maier BF, Brockmann D. Effective containment explains subexponential growth in recent confirmed COVID-19 cases in China. Science 2020;368:abb4557

4 Chinazzi M, Davis JT, Ajelli M, et al. The effect of travel restrictions on the spread of the 2019 novel coronavirus (COVID-19) outbreak. Science 2020;368:aba9757

5 Fang $\mathrm{H}$, Wang L, Yang Y. Human mobility restrictions and the spread of the novel coronavirus (2019-nCoV) in China. NBER Working Paper 2020:w26906.

6 The Economist. Not even Singapore has been able to avoid a lockdown. Economist 2020.

7 Carroll A. Lesson from Singapore: why we may need to think bigger. New York Times 2020.

8 Acemoglu D, García-Jimeno C, Robinson JA. State capacity and economic development: a network approach. Am Economic Rev 2015;105:2364-409.

9 Acemoglu D, Moscona J, Robinson JA. And American technology: evidence from the nineteenth century. AER 2016;106:p20161071.

10 Fukuyama F. Nous allons revenir un libéralisme des années 19501960. Le Point 2020.

11 Kermack WO, McKendrick AG. A contribution to the mathematical theory of epidemics. Proceedings of the Royal Society of London. series a, containing papers of a mathematical and physical character $1927 ; 115$.

12 Ferguson N, Laydon D, Nedjati Gilani G, et al. Report 9: impact of non-pharmaceutical interventions (NPIs) to reduce COVID19 mortality and healthcare demand 2020;77482.

13 Dispersing COVID-19 patients in Wuhan to other cities for better treatment. Available: http://liuyan.people.com.cn/threads/content? tid=6933339

14 Eve F. China's reaction to the coronavirus outbreak violates human rights. The Guardian 2020. 